\title{
Low Rate of Dysplasia Detection in Mucosa Surrounding Dysplastic Lesions in Patients Undergoing Surveillance for Inflammatory Bowel Diseases
}

\author{
Joren R. ten Hove, ${ }^{*}$ Erik Mooiweer, ${ }^{*}$ Evelien Dekker, ${ }^{\ddagger}$ Andrea E. van der Meulen-de Jong, ${ }^{\S}$ \\ G. Johan A. Offerhaus, ${ }^{\|}$Cyriel Y. Ponsioen, ${ }^{\ddagger}$ Peter D. Siersema, ${ }^{*, \oplus}$ and Bas Oldenburg ${ }^{*}$
}

${ }^{*}$ Department of Gastroenterology and Hepatology, University Medical Center Utrecht, Utrecht, The Netherlands; ${ }^{\S}$ Department of Gastroenterology and Hepatology, Leiden University Medical Center, Leiden, The Netherlands; ${ }^{\ddagger}$ Department of

Gastroenterology and Hepatology, Amsterdam Medical Center, Amsterdam, The Netherlands; "Department of Pathology,

University Medical Center Utrecht, Utrecht, The Netherlands; and "Department of Gastroenterology and Hepatology, Radboud University Medical Center, Utrecht, Nijmegen, The Netherlands

BACKGROUND \& AIMS: $\quad$ When dysplastic lesions are encountered during surveillance colonoscopy of patients with inflammatory bowel disease (IBD), guidelines recommend collection of additional biopsies from the surrounding mucosa to ensure the lesion has been adequately circumscribed. We aimed to determine the rate of dysplasia in mucosa biopsies collected from tissues surrounding dysplastic lesions during IBD surveillance.

METHODS:

In a retrospective study, we collected endoscopy and pathology reports from 1065 patients undergoing colonoscopic surveillance for IBD from 2000 through 2015 at 3 centers in the Netherlands. We analyzed reports from all patients with dysplastic lesions from whom biopsies of surrounding mucosa were collected. Among 194 patients with 1 or more visible dysplastic lesions, mucosal biopsies were collected from tissues adjacent to 140 dysplastic lesions from 71 patients $(63 \%$ male; $48 \%$ with ulcerative colitis, $42 \%$ with Crohn's disease, and $10 \%$ with indeterminate colitis).

RESULTS:

The mean number of surrounding mucosa biopsies collected per lesion was 3.4 (range, 1-6). Dysplasia was detected in 7 biopsies surrounding 140 areas of dysplasia (5.0\%) and 5 biopsies surrounding 136 areas of low-grade dysplasia (3.7\%). Dysplasia in biopsies of surrounding mucosa could be observed during 5 of 87 white light endoscopies and during 2 of 53 chromoendoscopies. In patients with dysplasia in mucosa surrounding lesions of low-grade dysplasia, post-resection surveillance did not reveal high-grade dysplasia or colorectal cancer.

CONCLUSIONS:

Dysplasia is detected in only $5 \%$ of biopsies collected from mucosa surrounding dysplastic lesions. This observation indicates that endoscopists accurately delineate the borders of dysplastic lesions during surveillance of patients with IBD. The lack of clinical consequences from routinely collecting biopsies from areas surrounding dysplastic lesions casts doubt on the usefulness and cost-effectiveness of this practice.

Keywords: Crohn's Disease; Ulcerative Colitis; Low-grade Dysplasia; Colorectal Cancer; Colon Cancer Surveillance.

Datients with inflammatory bowel disease (IBD) have an increased risk of colorectal cancer (CRC). ${ }^{1,2}$ Surveillance colonoscopies aimed at the detection and removal of dysplastic lesions, thereby preventing cancer and CRC-related mortality, are recommended to reduce this risk. ${ }^{3}$ Some guidelines still advise to take 4-quadrant random biopsies every $10 \mathrm{~cm}$ along the colonic mucosa, but the introduction of current state-of-the-art endoscopic equipment ${ }^{4}$ and the use of mucosal dye spraying ${ }^{5}$ may have rendered this practice redundant.
Whenever a dysplastic lesion is encountered during a surveillance colonoscopy, the management strategy for removal and follow-up is guided by several factors

Abbreviations used in this paper: CD, Crohn's disease; CRC, colorectal cancer; HD, high definition; HGD, high-grade dysplasia; IBD, inflammatory bowel disease; LGD, low-grade dysplasia; SD, standard definition; UC, ulcerative colitis; WLE, white light endoscopy.

(Q) Most current article

(C) 2017 by the AGA Institute $1542-3565 / \$ 36.00$

http://dx.doi.org/10.1016/j.cgh.2016.08.035 
including the morphology of the lesion, grade of dysplasia, and presence or absence of neoplasia in the mucosa surrounding the lesion. ${ }^{6}$ Most guidelines on IBD surveillance state that all visible lesions suspected of being neoplastic should be endoscopically removed if possible. In addition, biopsies should be taken from the mucosa surrounding the lesion. ${ }^{7-10}$ These biopsies should be negative for dysplasia to refrain from performing a colectomy (Supplementary Table 1). However, it can be questioned whether biopsies of surrounding mucosa are still needed in this era of high-definition (HD) endoscopes and chromoendoscopy.

The aim of the present study was to assess the dysplasia yield from biopsies of surrounding mucosa. In addition, we aimed to study the clinical consequences of these biopsies in the setting of surveillance colonoscopies in IBD.

\section{Methods}

\section{Patient Selection}

Patients undergoing colonoscopic surveillance for IBD between January 2000 and January 2015 were identified from 3 Dutch tertiary referral centers by using diagnosis treatment combinations for IBD in the respective hospital databases. The diagnosis treatment combinations are in many ways analogous to the World Health Organization International Classification of Disease coding system. Only colonoscopies in which a random biopsy protocol or pancolonic dye spraying was used were considered surveillance procedures and included in this study. From 2010 onwards, chromoendoscopy has been gradually incorporated as the standard of care for IBD surveillance in the participating centers. The patients' medical records were reviewed to retrieve demographic data, IBD type, date of IBD diagnosis, maximum endoscopic disease extent, family history of CRC, and a history of prior dysplasia before the studied surveillance period.

\section{Identification of Dysplastic Lesions}

For the observed study period all endoscopy and pathology reports were manually screened for the occurrence of colonic dysplasia. For each dysplastic lesion, location, size, morphology, and grade of dysplasia were documented. Morphology of the dysplastic lesion was categorized as polypoid or non-polypoid on the basis of the endoscopic description. Lesions described as sessile or pedunculated polyps and Paris type 0-1 lesions were classified as polypoid. Lesions labeled as flat, non-polypoid, or as mucosal irregularities were considered non-polypoid. Histopathologic grade of dysplasia was categorized as low-grade dysplasia (LGD), high-grade dysplasia (HGD), or colorectal cancer (CRC). ${ }^{11}$ Lesions described in the pathology report as indefinite for dysplasia were excluded. Dysplastic lesions accompanied by LGD in spatially distinct mucosa on random biopsies or LGD originating from other targeted lesions during the same colonoscopic procedure were categorized as multifocal dysplasia. The extent and grade of endoscopic inflammation were recorded, and endoscopy and pathology reports were reviewed for the presence of biopsies taken in direct proximity to the lesion. Biopsies were classified as biopsies of surrounding mucosa if both the endoscopy and pathology reports provided biopsy results unequivocally traceable to a single dysplastic lesion. Biopsies described as taken directly from the outer margin of the lesions or biopsies taken from a polypectomy scar were excluded. For each lesion, the number of individual biopsy specimens was retrieved from the pathology report. If revision of a biopsy was performed, the revised conclusion was used in the analyses.

\section{Statistical Analysis}

Baseline demographic data are presented for unique patients rather than lesions. Dichotomous outcomes are presented as the number of events with corresponding percentage and were compared by using the $\chi^{2}$ method or Fisher exact test where appropriate. Continuous data are presented as means with standard deviation or median and range and were compared by the Student $t$ test or Mann-Whitney $U$ test according to normality. The $P$ value for identification of a statistically significant difference was set at .05. All data analyses were performed by using SPSS version 21 (IBM Corp, Armonk, NY).

\section{Results}

\section{Patients}

In total, 1065 IBD patients were enrolled in surveillance programs during the study period. In 196 patients (18\%) a visible dysplastic lesion was found during surveillance within the studied period. For 140 dysplastic lesions (71 patients), additional biopsies of surrounding mucosa were taken (Figure 1). Clinical characteristics of the patients are displayed in Table 1 and Supplementary Table 2. The majority of patients were male $(63.4 \%)$. Thirty-four patients (48\%) had ulcerative colitis (UC), 30 patients (42\%) had Crohn's disease (CD), and 7 patients $(10 \%)$ had indeterminate colitis. Mean age at the time of IBD diagnosis was $35 \pm 13$ years, and mean age at the time of dysplasia diagnosis was $56 \pm 12$ years.

\section{Lesions With Biopsies of Surrounding Mucosa}

Table 2 displays results of the per-lesion analysis for the included cases. Lesions with biopsies of surrounding mucosa had a mean size of $5.5 \mathrm{~mm}$, and the majority had polypoid morphology (82\%). The mean number of biopsies of surrounding mucosa per lesion was 3.3 (range, 1-6). In 83 cases (60\%) multifocal LGD was found. 


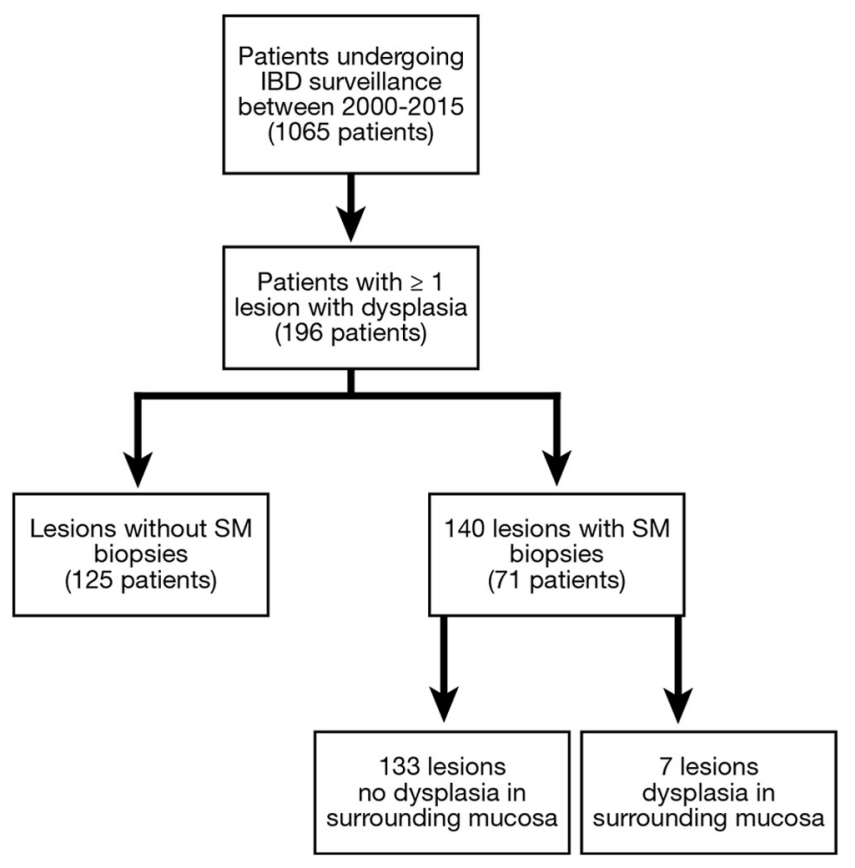

Figure 1. Flowchart for selection of cases.

Ninety-eight lesions (70\%) were removed endoscopically and 12 lesions (9\%) by using biopsy forceps, whereas 30 lesions $(21 \%)$ were initially only biopsied.

\section{Dysplasia Yield From Biopsies of Surrounding Mucosa}

Dysplasia was detected in surrounding mucosa biopsies in 7 of 140 neoplastic lesions (5.0\%). The rate of dysplasia per biopsy was 7 of 492 biopsies (1.4\%). The 7 lesions with surrounding mucosa dysplasia were

Table 1. Baseline Characteristics for Included Patients

\begin{tabular}{lc}
\hline & $\mathrm{n}(\%)$ \\
\hline No. of patients & 71 \\
Male gender (\%) & $45(63.4)$ \\
UC & 34 \\
Extensive colitis & $9(26.4)$ \\
Left-sided & $24(70.6)$ \\
Unknown & $1(2.9)$ \\
Crohn's colitis & 30 \\
Segmental colitis $>50 \%$ & $13(43.3)$ \\
Segmental colitis $<50 \%$ & $15(50.0)$ \\
Unknown & $2(6.7)$ \\
Indeterminate colitis & 7 \\
Segmental colitis $>50 \%$ & $1(14.3)$ \\
Segmental colitis $<50 \%$ & $5(71.4)$ \\
Unknown & $1(14.3)$ \\
Age at first dysplasia detection, & $56.0 \pm 11.9$ \\
$\quad y$ (mean \pm standard deviation) & \\
Age at IBD diagnosis, $y$ & $34.6 \pm 13.1$ \\
$\quad$ (mean \pm standard deviation) & \\
History of dysplasia (before surveillance) & $10(14.1)$ \\
Postinflammatory polyps & $12(17.1)$ \\
Primary sclerosing cholangitis & $4(5.7)$ \\
&
\end{tabular}

larger than the lesions without surrounding mucosa dysplasia, although this difference was not statistically significant ( $8.8 \mathrm{~mm}$ vs $5.3 \mathrm{~mm}, P=.21$ ). Morphology, site, multifocality, histology, and technique used were not significantly different between the groups. Characteristics of the individual cases that were positive for surrounding mucosa dysplasia are displayed in Table 3. Surrounding mucosa dysplasia rates were not found to change over time. Surrounding mucosa dysplasia was detected in 3 of 70 lesions in colonoscopies performed before October 2010 and in 4 of 70 lesions detected later on $(P=.50)$.

\section{Dysplasia Yield From Biopsies of Surrounding Mucosa and Grade of Dysplasia of Colonic Lesions}

Four lesions with HGD were accompanied by biopsies of surrounding mucosa, 2 of which were positive for surrounding mucosa dysplasia. Both patients underwent colectomy shortly after diagnosis. Five of 136 LGDcontaining lesions $(3.7 \%)$ were found to have dysplasia in biopsies of surrounding mucosa. Mean time until the first follow-up endoscopy procedure was 7.8 months, and the mean number of total endoscopic follow-up endoscopies was 5.5. Among patients with LGD lesions and 1 or more positive biopsies of surrounding mucosa, no occurrence of HGD or CRC was observed on follow-up surveillance endoscopy during a median follow-up time of 37 months (Table 3).

\section{Discussion}

This study evaluated the value of taking biopsies from mucosa surrounding dysplastic lesions during surveillance colonoscopies in patients with longstanding IBD in the colon. Within this retrospective cohort, 196 patients had at least 1 visible dysplastic lesion, whereas biopsies of surrounding mucosa were taken in only 71 patients. The dysplasia yield of these biopsies was 7 per 140 lesions (5\%) and decreased to 5 per 136 lesions (3.7\%) when only lesions containing LGD were analyzed. The two patients with HGD and surrounding mucosa dysplasia were treated surgically. In patients with LGD lesions and surrounding mucosa dysplasia, colectomy was not performed, and a more intensive surveillance schedule was instigated. HGD or cancer was found in none of these patients during follow-up thus far.

Resection and/or intensified surveillance is considered to be the strategy of choice for dysplastic lesions in patients with IBD of the colon. ${ }^{12,13}$ Guidelines recommend taking additional biopsies from mucosa surrounding these lesions to ensure complete removal of the lesion. However, this practice is not based on solid evidence, and the impact on patient outcomes has not been assessed. ${ }^{7-10}$ Furthermore, no consensus exists on the number of biopsies and the optimal distance from the biopsy to the 
Table 2. Per-Lesion Analysis: Cases With SM Biopsies

\begin{tabular}{|c|c|c|c|c|}
\hline & Total & SM negative for dysplasia & SM positive for dysplasia & $P$ value \\
\hline No. of lesions & 140 & 133 & 7 & - \\
\hline No. of SM biopsies & 3.4 & 3.3 & 3.7 & .36 \\
\hline Lesion size, mm (mean) & 5.5 & 5.3 & 8.8 & .21 \\
\hline \multicolumn{5}{|l|}{ Morphology } \\
\hline Polypoid & $115(82.1 \%)$ & $111(83.5 \%)$ & $4(57.1 \%)$ & \multirow[t]{2}{*}{.21} \\
\hline Non-polypoid & 25 (17.9\%) & 22 (16.5\%) & $3(42.9 \%)$ & \\
\hline \multicolumn{5}{|l|}{ Histologic architecture } \\
\hline Adenomatous & $108(77.2 \%)$ & $104(78.2 \%)$ & $4(57.1 \%)$ & \multirow[t]{3}{*}{.08} \\
\hline Serrated & $10(7.1 \%)$ & $10(7.5 \%)$ & - & \\
\hline Other/colitis-associated & $22(15.7 \%)$ & $19(14.3 \%)$ & $3(42.9 \%)$ & \\
\hline \multicolumn{5}{|l|}{ Location of lesion } \\
\hline Left colon & 55 (39.3\%) & $53(39.8 \%)$ & $2(28.6 \%)$ & \multirow[t]{3}{*}{.83} \\
\hline Transverse colon & $32(22.9 \%)$ & $30(22.6 \%)$ & $2(28.6 \%)$ & \\
\hline Right colon & $53(37.9 \%)$ & $50(37.6 \%)$ & $3(42.9 \%)$ & \\
\hline Multifocal dysplasia & $84(60.0 \%)$ & $81(60.9 \%)$ & $3(42.9 \%)$ & .49 \\
\hline \multicolumn{5}{|l|}{ Lesion management: } \\
\hline Endoscopic removal & $98(70 \%)$ & $95(71.4 \%)$ & $3(42.9 \%)$ & \multirow[t]{3}{*}{.11} \\
\hline Forceps removal & $12(9 \%)$ & $10(7.5 \%)$ & $2(28.6 \%)$ & \\
\hline Initially only biopsied & $30(21 \%)$ & $28(21.1 \%)$ & $2(28.6 \%)$ & \\
\hline \multicolumn{5}{|l|}{ Endoscopic technique used } \\
\hline Chromoendoscopy & $53(37.9 \%)$ & $51(38.3 \%)$ & $2(28.6 \%)$ & \multirow[t]{3}{*}{.78} \\
\hline SD-WLE & $44(31.4 \%)$ & $42(31.6 \%)$ & $2(28.6 \%)$ & \\
\hline HD-WLE & $43(30.7 \%)$ & $40(30.1 \%)$ & $3(42.9 \%)$ & \\
\hline
\end{tabular}

margin of the lesion or polypectomy site. Variations in these factors will undoubtedly impact the yield from biopsies of surrounding mucosa. In addition, these biopsies may guide clinical decision-making by confirming active or previous inflammation and thereby support the diagnosis of colitis-associated dysplasia. The relevance of this point lies in the fact that colitis-associated dysplasia is thought to progress more rapidly to advanced dysplasia than conventional adenomatous lesions. ${ }^{14,15}$ The distinction between sporadic or colitis-associated dysplasia is hampered by the absence of unequivocal histopathologic and endoscopic criteria but can be aided by the confirmation of present or previous inflammation, as provided by biopsies of surrounding mucosa. ${ }^{16}$

Because no previous studies on the dysplasia yield from biopsies of surrounding mucosa are available, one might compare the results from our study with those from random biopsy protocol studies. Van den Broek et al ${ }^{17}$ performed a retrospective analysis of 466 surveillance colonoscopies and found a neoplasia yield of $5.7 \%$ per colonoscopy for random biopsies. In the per-lesion analysis, only 24 random biopsies $(0.2 \%)$ were found to contain dysplasia. This study supported the hypothesis of safely omitting a random biopsy protocol because of lack of clinical impact. However, the best way of putting our data into perspective would be a comparison with the dysplasia yield of random biopsies from procedures in which concurrent visible dysplastic lesions were identified. When analyzing dysplasia yields stratified for the detection method in a per-colonoscopy analysis, the above mentioned study reported 7 colonoscopies with dysplasia in both random and targeted biopsies out of a total of 83 colonoscopies with at least 1 targeted dysplastic lesion $(8.4 \%)$.

Because chromoendoscopy with targeted biopsies became the preferred surveillance method during the study period, white light endoscopy (WLE) (standard definition [SD] and high definition [HD]) was gradually replaced by pancolonic dye spraying in all participating centers. Cases of surrounding mucosa dysplasia were found in SD WLE, HD WLE, and chromoendoscopy procedures (Supplementary Figure 1). HD colonoscopes, supported by the use of dye spraying, are expected to improve the detection of dysplasia and allow for a better assessment of the lesion once detected. On the other hand, more indistinct lesions may have been missed by using one of these techniques, especially if SD and not HD equipment was used, which might have led to selection bias. The small number of positive cases precludes statistically sound conclusions on this matter.

The most straightforward explanation for positive biopsies of surrounding mucosa is a limited visibility of the lesion borders (before resection) leading to residual dysplastic tissue (after resection), as displayed in Figure 2. Less distinct lesion borders may complicate both the selection of the snare size and shape as well as placement of a snare or other devices used in endoscopic removal. Taking biopsies of surrounding mucosa will offer the endoscopist a chance of detecting residual dysplasia, which might be further concealed by hemorrhage or cauterization after resection. However, even when the margin of a lesion can be clearly distinguished and the resection is likely to be complete, histologic assessment of the surrounding mucosa is considered standard practice. 


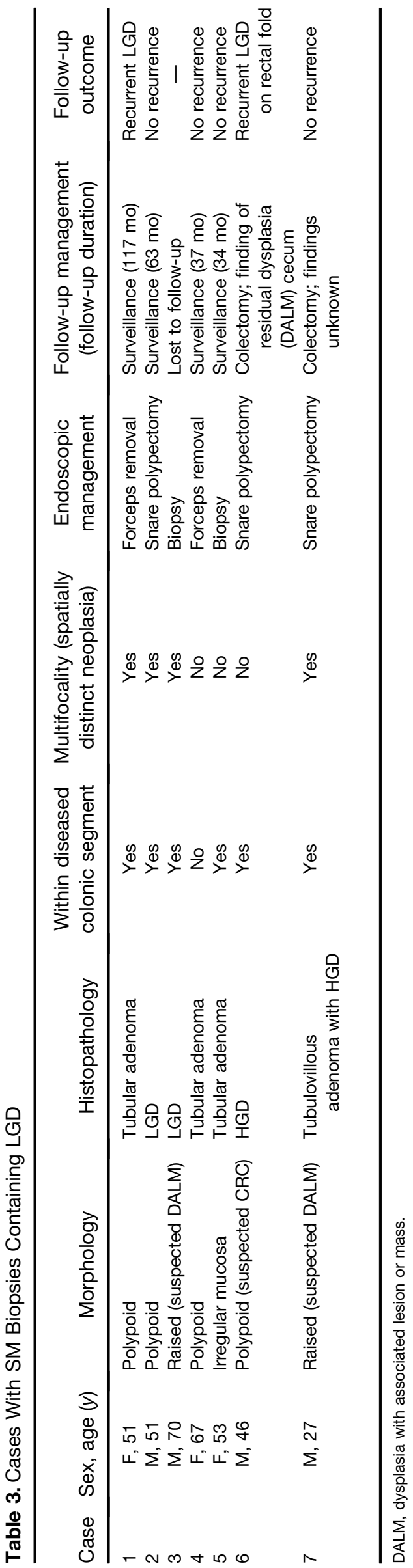

There are currently no directives for the separate use of specific biopsy equipment for surrounding mucosa. It is unknown whether using the same biopsy forceps for random, semi-random, and targeted biopsies in the same session may cause residual tissue of one lesion to be incorrectly allocated to another lesion.

Endoscopic removal of lesions in areas affected by colitis pose a major challenge to endoscopists, and careful assessment before endoscopic resection is critical. In particular, scarring of the mucosa may lead to a piecemeal resection, which increases the risk of residual neoplastic tissue at the resection site. Whether ablative therapies such as argon plasma coagulation or soft coagulation (using a snare tip) can safely be used in the prevention of neoplasia recurrence in patients with IBD remains to be seen. ${ }^{18}$

It is important to note that our overall analysis included visible lesions containing HGD. Colectomy is considered to be the standard treatment for invisible HGD because high rates of CRC have been reported in these patients. However, one report concluded that visible polypoid lesions containing HGD could be treated by endoscopic resection and close follow-up without surgical intervention. ${ }^{19}$ In the present study, the majority of cases consisted of LGD lesions. Although they were infrequently detected, visible HGD lesions showed surrounding mucosa dysplasia in 2 cases. This suggests a higher risk of incomplete resection for these more advanced lesions, although our study was not designed to test this hypothesis. Currently, the optimal management of HGD in a lesion that is endoscopically visible remains ill-defined, in part because data on the risk of cancer after endoscopic management are lacking.

Strengths of this study include the fact that a large cohort was used to identify cases. In addition, endoscopies were performed in referral centers by experienced endoscopists who used state-of-the-art endoscopic equipment. Our cohort consists of patients eligible for IBD surveillance regardless of the specific IBD subtype (UC, CD, or indeterminate colitis), which is in contrast to some earlier studies in which only patients with UC were included.

Our study also has some limitations. First, because this is a retrospective study, there were no standard operating procedures for sampling the surrounding mucosa. Second, biopsies of surrounding mucosa were only available for a subgroup of dysplastic lesions, and the physicians' considerations to refrain from taking these additional biopsies could not be ascertained. It is possible that positive cases were missed because of misjudging of the apparent need for biopsies of surrounding mucosa, for example, if the endoscopic appearance resembles that of a sporadic lesion or if the lesion is found outside of an area visibly affected by inflammation. Also, a lesion that is not wellcircumscribed may lower the threshold for taking additional biopsies. However, we expect that this selection bias would have led to an increase in the rate of dysplasia, because the endoscopist would have been more 
Figure 2. Schematic representation of taking biopsies of SM after polypectomy. Explicit recommendations on number of biopsies, distance of biopsies to resection site, and timing of biopsies (before or after resection) are lacking.
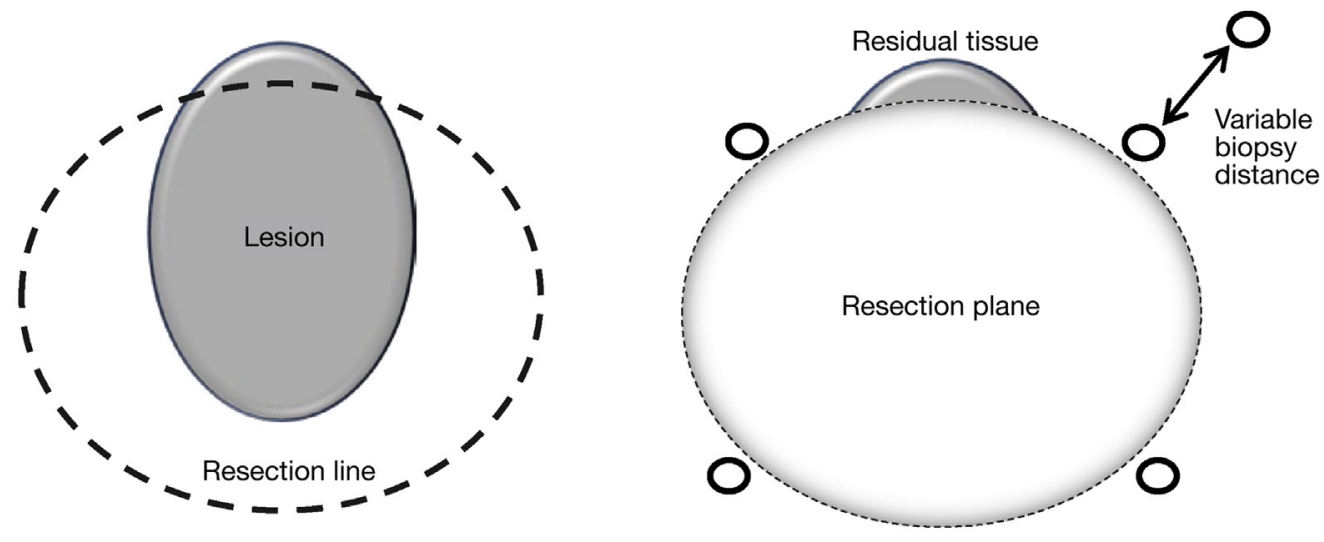

frequently inclined to take biopsies of surrounding mucosa in case of indistinct lesion borders. Nonetheless, the number of cases in which this specific guideline recommendation is adhered to is strikingly low.

We chose not to include biopsies of mucosa surrounding targeted lesions that turned out to be non-dysplastic, which would have decreased the relative number of positive biopsies in this study. In addition, the retrospective nature of our study precluded systematic categorization of lesions and the exact technique of lesion removal. Moreover, not all lesions were directly removed endoscopically after taking biopsies of surrounding mucosa, which makes the interpretation of the subsequent disease course more difficult.

In summary, this study evaluated the rate of dysplasia in biopsies routinely taken from mucosa surrounding dysplastic lesions in patients undergoing IBD surveillance. The rate of surrounding mucosa dysplasia was $5.0 \%$ per lesion, which suggests that the general ability to demarcate lesion margins is good. When our results are confirmed in a larger and prospective setting, the current practice of routinely taking biopsies of surrounding mucosa may become redundant. The increasing use of HD equipment, aided by advanced imaging techniques, will likely continue to enhance visibility and reduce the need to routinely take these additional biopsies. Conversely, when relied on too heavily, negative biopsies of surrounding mucosa may lead to a false sense of security if other endoscopic features and patient characteristics are overlooked. Factors other than the presence or absence of dysplasia should be included in a consideration toward more selective use of these biopsies. Specifically, making a distinction between sporadic and colitis-associated dysplasia solely on the basis of endoscopic features can be problematic. In any case, pre-removal assessment should include thorough cleaning of the lesion and surrounding mucosa. In addition, targeted chromoendoscopy as well as digital image enhancing techniques can aid in the proper identification of the lesion and its borders.

The lack of clinical consequences related to taking biopsies of surrounding mucosa casts doubt on the usefulness and cost-effectiveness of this practice as a routine measure.

\section{Supplementary Material}

Note: To access the supplementary material accompanying this article, visit the online version of Clinical Gastroenterology and Hepatology at www.cghjournal.org, and at http://dx.doi.org/10.1016/j.cgh.2016.08.035.

\section{References}

1. Eaden JA, Abrams KR, Mayberry JF. The risk of colorectal cancer in ulcerative colitis: a meta-analysis. Gut 2001; 48:526-535.

2. Bernstein CN, Blanchard JF, Kliewer E, et al. Cancer risk in patients with inflammatory bowel disease: a population-based study. Cancer 2001;91:854-862.

3. Laine L, Kaltenbach T, Barkun A, et al. SCENIC international consensus statement on surveillance and management of dysplasia in inflammatory bowel disease. Gastroenterology 2015;148:639-651.e28.

4. Subramanian V, Ramappa V, Telakis E, et al. Comparison of high definition with standard white light endoscopy for detection of dysplastic lesions during surveillance colonoscopy in patients with colonic inflammatory bowel disease. Inflamm Bowel Dis 2013;19:350-355.

5. Subramanian V, Mannath J, Ragunath K, et al. Meta-analysis: the diagnostic yield of chromoendoscopy for detecting dysplasia in patients with colonic inflammatory bowel disease. Aliment Pharmacol Ther 2011;33:304-312.

6. Rutter MD, Riddell $\mathrm{RH}$. Colorectal dysplasia in inflammatory bowel disease: a clinicopathologic perspective. Clin Gastroenterol Hepatol 2014;12:359-367.

7. Cairns SR, Scholefield JH, Steele RJ, et al. Guidelines for colorectal cancer screening and surveillance in moderate and high risk groups (update from 2002). Gut 2010;59:666-689.

8. Kornbluth A, Sachar DB. Ulcerative colitis practice guidelines in adults: American College of Gastroenterology, Practice Parameters Committee. Am J Gastroenterol 2010;105:501-523.

9. Marion JF, Sands BE. The SCENIC consensus statement on surveillance and management of dysplasia in inflammatory bowel disease: praise and words of caution. Gastroenterology 2015;148:462-467. 
10. Annese V, Daperno M, Rutter MD, et al. European evidence based consensus for endoscopy in inflammatory bowel disease. J Crohns Colitis 2013;7:982-1018.

11. Riddell RH, Goldman H, Ransohoff DF, et al. Dysplasia in inflammatory bowel disease: standardized classification with provisional clinical applications. Hum Pathol 1983;14:931-968.

12. Wanders LK, Dekker E, Pullens B, et al. Cancer risk after resection of polypoid dysplasia in patients with longstanding ulcerative colitis: a meta-analysis. Clin Gastroenterol Hepatol 2014;12:756-764.

13. Rubin PH, Friedman S, Harpaz N, et al. Colonoscopic polypectomy in chronic colitis: conservative management after endoscopic resection of dysplastic polyps. Gastroenterology 1999;117:1295-1300.

14. Itzkowitz S. Colon carcinogenesis in inflammatory bowel disease: applying molecular genetics to clinical practice. J Clin Gastroenterol 36:S70-S74, discussion S94-S96.

15. Choi C-HR, Ignjatovic-Wilson A, Askari A, et al. Low-grade dysplasia in ulcerative colitis: risk factors for developing high-grade dysplasia or colorectal cancer. Am J Gastroenterol 2015; 110:1461-1471.

16. Bernstein CN. ALMs versus DALMs in ulcerative colitis: polypectomy or colectomy? Gastroenterology 1999;117:1488-1492.
17. van den Broek FJC, Stokkers PCF, Reitsma JB, et al. Random biopsies taken during colonoscopic surveillance of patients with longstanding ulcerative colitis: low yield and absence of clinical consequences. Am J Gastroenterol 2014;109:715-722.

18. East JE, Toyonaga T, Suzuki N. Endoscopic management of nonpolypoid colorectal lesions in colonic IBD. Gastrointest Endosc Clin N Am 2014;24:435-445.

19. Blonski W, Kundu R, Furth EF, et al. High-grade dysplastic adenoma-like mass lesions are not an indication for colectomy in patients with ulcerative colitis. Scand J Gastroenterol 2008; 43:817-820.

\section{Reprint requests}

Address requests for reprints to: Bas Oldenburg, MD, PhD, Department of Gastroenterology and Hepatology, University Medical Center Utrecht, Heidelberglaan 100, 3584 CX Utrecht, The Netherlands. e-mail: b.oldenburg@ umcutrecht.nl; fax: (0031) 887555533.

\section{Conflicts of interest}

This author discloses the following: Bas Oldenburg is a consultant for AbbVie BV, MSD BV, and Ferring BV. The remaining authors disclose no conflicts.

\section{Funding}

Supported by an unrestricted grant from MSD BV and an unrestricted grant from Ferring BV. 


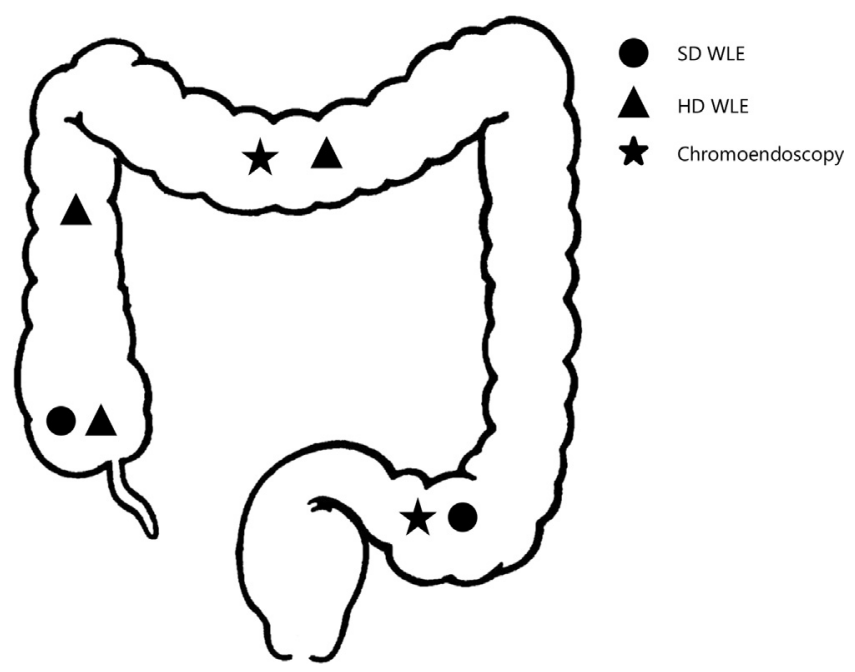

Supplementary Figure 1. Location of lesions with positive SM biopsies, according to endoscopic technique used to detect lesion.

Supplementary Table 1. Excerpts of Guidelines Regarding Sampling of SM

BSG guideline (update 2010)

SCENIC statement; joint American Gastroenterological Association \& American Society for Gastrointestinal Endoscopy consensus (2015) ${ }^{3}$

ECCO statement $(2013)^{10}$

American College of Gastroenterology $(2010)^{8}$
"It is essential to biopsy the flat mucosa surrounding any dysplastic polyp to assess the extent of disease (as it may not be apparent macroscopically) and also to assess whether there is any dysplasia in the surrounding flat mucosa. If a dysplastic polyp occurs in an area proximal to the microscopic level of inflammation, with no dysplasia in flat mucosa, it can be regarded as a sporadic adenoma and treated accordingly."

"The term endoscopically resectable indicates that distinct margins of the lesion could be identified, the lesion appears to be completely removed on visual inspection after endoscopic resection, histologic examination of the resected specimen is consistent with complete removal, and biopsy specimens taken from mucosa immediately adjacent to the resection site are free of dysplasia on histologic examination."

"If the polypectomy is confirmed complete by histology, biopsies obtained from the flat mucosa immediately adjacent to the polypectomy site show no dysplasia and no dysplasia is found elsewhere in the colon, a careful colonoscopic follow-up preferably with chromoendoscopy at 3 months before reverting to annual surveillance is recommended, because at least half of such patients may develop further lesions.

"If the lesion is not resectable, or is associated with dysplasia in the adjacent mucosa, then colectomy is indicated due to the high risk of concomitant CRC."

"If the lesion is resected in its entirety by colonoscopic polypectomy and if no dysplasia is found in the adjacent flat mucosa or anywhere else in the colon, long-term follow-up has not found an increased risk of cancer in these cases, suggesting that vigilant follow-up surveillance colonoscopy may suffice." 
Supplementary Table 2. Baseline Characteristics for All Patients With $\geq 1$ Lesions Containing Dysplasia

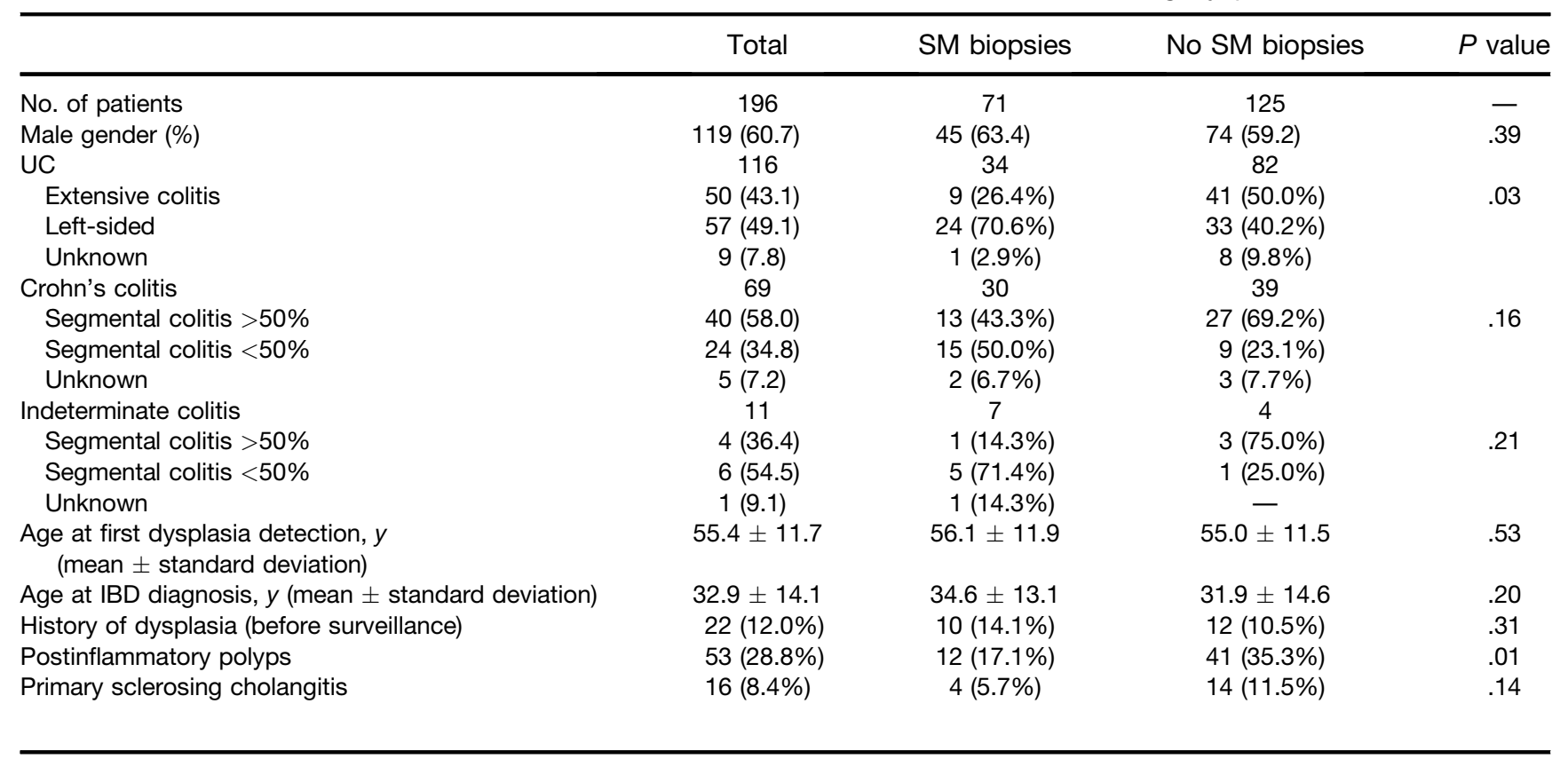

